\title{
Susac Syndrome a Case Report
}

Abdelfattah Aljalil ${ }^{1 *}$, Mohamed Amine Hanine ${ }^{1}$, Mohamed Elakhiri ${ }^{1}$, Mohamed Badaoui ${ }^{2}$, Shamil Louaya ${ }^{3}$, Youssef Darouassi ${ }^{1}$, Haddou Ammar ${ }^{1}$

\footnotetext{
${ }^{1}$ Department of Otorhinolaryngology Head and Neck, Avicenna military hospital, Marrakech, Morocco

${ }^{2}$ Department of Internal Medicine, 1CMC Agadir, Morocco

${ }^{3}$ Department of Ophthalmology, 1CMC Agadir, Morocco
}

DOI: $10.36347 /$ sjmcr.2020.v08i03.015

| Received: 27.02.2020 | Accepted: 05.03.2020 | Published: 13.03.2020

*Corresponding author: Abdelfattah Aljalil

Abstract

Susac syndrome or "SICRET syndrome" is a recent, rare entity, defined by a diagnostic triad grouping together neurological, ophthalmological and inner ear damage. Its physiopathology is still poorly understood and its treatment is not yet codified. We report the case of a female 34-year-old patient who presented with a Susac syndrome which developed well under medical treatment with corticosteroids and we recall all clinical, evolutive and therapeutic features of this rare syndrome.

Keywords: Susac Syndrome evolutive.

Copyright @ 2020: This is an open-access article distributed under the terms of the Creative Commons Attribution license which permits unrestricted use, distribution, and reproduction in any medium for non-commercial use (NonCommercial, or CC-BY-NC) provided the original author and source are credited.

\section{INTRODUCTION}

Susac syndrome is a rare microangiopathy characterized by triple damage to the brain, retina and inner ear. It was first described in 1973 by Pfaffenbach and Hollenhorst [1]. It was in 1979 when Susac and al. gave the name of "SICRET syndrome" (small infarctions of cochlear, retinal and encephalic tissue) to this entity [2]. Since then, few over 200 cases have been reported in the literature [3]. Here we report the case of a patient with Susac syndrome.

\section{ObSERVATION}

A female 34-year-old patient, without particular pathological history or cardiovascular risk factors, was referred to our service by her ophthalmologist, who follows her for bilateral scotomas, for disabling vertigo associated with progressive left hearing loss and recent appearance of right hearing loss. In addition, the patient is followed up in the neurology department for language and memory disorders evolving for 6 years.

The clinical examination found a patient who was conscious and showed no focal neurological signs. The ophthalmological examination revealed a bilateral amputation of the visual fields on the periphery and an obliteration of the branches of the central retinal artery (CRA) at angiography (fig1).

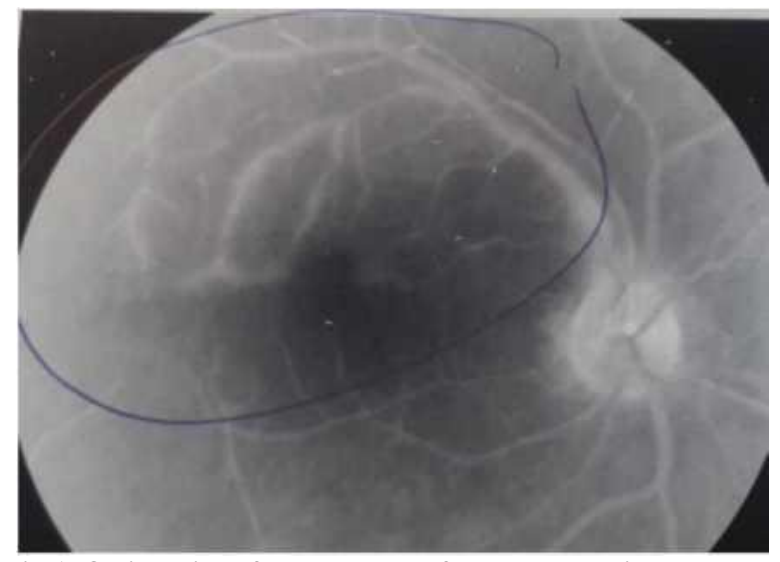

Fig-1: Obliteration of the branches of the central retinal artery at angiography

The otorhinolaryngological examination found a left peripheral vestibular syndrome with a bilateral hearing loss predominant on the low frequencies at the tonal audiometry (fig2).

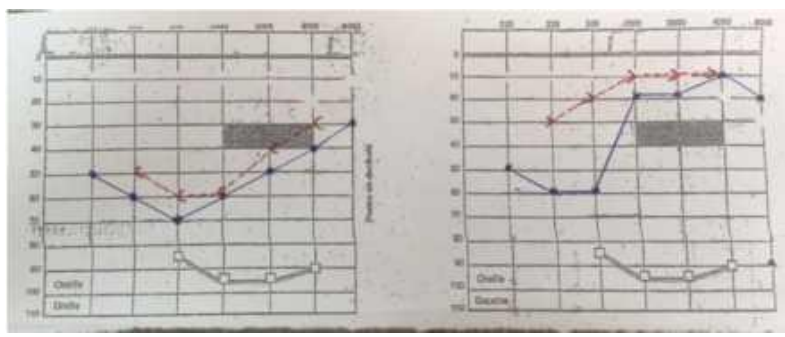

Fig-2: Audiogram showing bilateral mixt hearing loss 
Brain magnetic resonance imaging showed hyperintensity in the corpus callosum. The lumbar puncture brought back clear cerebrospinal fluid with a lymphocytic reaction.

\section{The immune status was negative.}

The diagnosis of Susac syndrome or SICRET syndrom was accepted in view of the clinical presentation and the MRI and angiography data.

The patient received bolus corticosteroid therapy $(1 \mathrm{~g} / \mathrm{d})$ combined with anti-platelet aggregation agents, anti-dizziness, vasodilators and oxygen therapy.

The development after one month was marked by a big improvement in vertigo as well as in hearing, especially on the left side (fig 3).

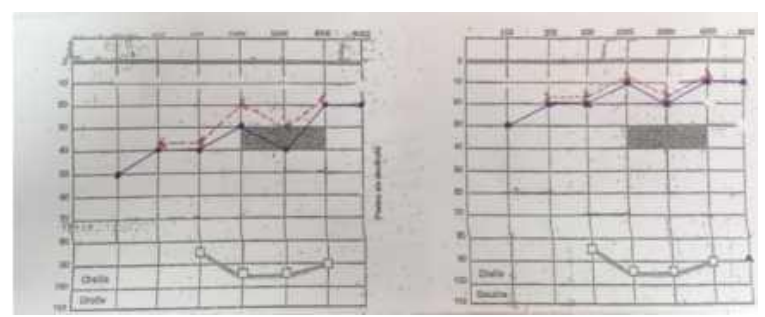

Fig-3: clear improvement of bilateral hearing loss

\section{DiscuSSION}

SUSAC syndrome or "SICRET syndrome" is a recent, rare entity defined by a diagnostic triad grouping together almost constant neurological damage associated with ophthalmological and inner ear damage [4].

This syndrome mainly affects women aged between 20 and 40 years with extremes ranging from 7 to 72 years [3]. The physiopathology is poorly understood. Several hypotheses have been put forward, in particular an autoimmune origin, hypercoagulability or even an embolic disease [4]. Brain and muscle biopsies have similarities to dermatomyositis. It is believed to be an autoimmune endotheliopathy where the antibodies against endothelial cells are responsible for micro-infarctions and perivascular inflammatory infiltrates of small vessels [5].

It is interesting to note that the three affected organs have vessels of similar structure: tightly junctioned endothelium vessels. This syndrome is defined by a diagnostic triad, however one of the disorders may be absent in the rough forms [4].

Neurological involvement is almost constant as it is present in $95 \%$ of cases during the beginning of the disease. This involvement is very variable, ranging from simple headaches to large neuro-psychiatric symptoms.
MRI, especially in T2 and FLAIR sequences, is contributive in $95 \%$ of cases and finds lesions in hyperintensity of the white matter, the gray matter and the leptomeninges, above and under tentorial. Lesions of corpus callosum are almost always presents [6, 7]. Cerebral atrophy is a sign of the severity and a negative development sign of the disease [4].

The lumbar puncture reveals a lymphocytic reaction and a moderate hyperproteinorachy. The most frequent ophthalmologic involvement is an occlusion of a branch of CRA, generally unilateral but which can become bilateral over time [5].

Other signs are less often reported because they are clinically asymptomatics. These are segmental occlusions and vasculitides of the arterial network [8]. Yellowish intravascular deposits are found, especially identifiable with AGF by their hyperfluorescence. These deposits, from different locations of arterial occlusions, could be either a sign preceding arterial occlusions or a sign of disease activity [9]. It has been proposed to introduce preventive corticosteroid therapy in the presence of these deposits. This early therapy would improve the prognosis [5]. Long-term follow-up is little reported in the literature.

The most common ear, nose and throat disorder is asymmetrical bilateral hearing loss, regularly associated with tinnitus. It mainly affects the low and medium frequencies as illustrated in our case. This deafness is linked to vascular damage to the apical portion of the cochlea [10]. Reaching higher frequencies is a sign of seriousness. In the long term, deafness and tinnitus are bothersome after-effects for these young women in their socio-professional life. Finally, dizziness is possible and reported more rarely. If for the majority of cases, the disease lasts less than two years during which symptoms can fluctuate, some cases have a polycyclic course over more than two years with phases of exacerbation interspersed with remission (our case) and other cases goes on to chronicity where symptoms can fluctuate over a period of more than two years without any remission [4-11]. Due to the rarity of the disease, its treatment remains not codified.

In 2007, Rennebohm and Susac [11] proposed therapeutic axes. These "recommendations" are taken from that of dermatomyositis due to its physiopathological relationship. When faced with an encephalopathy, it is advisable to start intravenous corticosteroid therapy with an oral relay combined with a monthly Ig treatment for six months. Cyclophosphamide and mofetil mycophenolate are reserved for severe cases such as plasmapheresis. The decrease in corticosteroid therapy will be done in very progressive steps. For patients without signs of encephalopathy, treatment may be less aggressive. Given the current impossibility of predicting the 
progression to severe neurological damage, the authors recommend an initial treatment combining corticosteroid therapy and Ig. The other therapies (antiaggregants, anticoagulants...). Have not been proven to be effective. Some authors suspect that the delay in the introduction of corticosteroid therapy is a penalizing factor in terms of ophthalmology [4].

\section{CONCLUSION}

SUSAC syndrome is a rare entity. Its diagnosis is based on a tripod with neurological, ophthalmological and ENT involvement. Its physiopathology remains uncertain, but the hypothesis of autoimmune disease prevails. Its multidisciplinary management must be early and is essentially based on corticosteroid therapy and immunosuppressants.

\section{REFERENCES}

1. Pfaffenbach DP, Hollenhorst RW Microangiopathy of the retina. JAMA. 1973; 225:480-3.

2. Susac JO, Hardman JM, Selhorts JB. Microangiopathy of the brain and retina. Neurology. 1979; 29:313-6.

3. Rennebohm R. Susac's Syndrome - Update. Journal of the Neurological Sciences.2010 ; 299 : 86-91.

4. Chehab H. El. Syndrome de SUSAC. Modes d'entrée et évolutions variables : à propos de deux cas. Journal français d'ophtalmologie.2010; 33, 575.e1-575.e7.

5. O'Halloran HS, Andrew Pearson A, Barry Lee W, Susac JO, Berger JR. Microangiopathy of the brain retina, and cochlea (Susac syndrome). A report of five cases and a review of the literature. Ophthalmology. 1998:105-6.

6. Susac JO, Egan RA, Rennebohm RM, Lubow M. Susac's $\quad$ syndrome: 1975-2005 microangiopathy/autoimmune endotheliopathy. JNS 2007;257:270-2.

7. Le Moigne F, Lamboley JL, Vitry T, El Chehab H, Guilloton L, Drouet A. Susac syndrome, retinocochleocerebral microangiopathy: contribution of MRI. J Neuroradiol. 2009;36(5):285-89.

8. Martinet N, Fardeau C, Adam R, Bodaghi B, Papo T, Piette JC. Fluorescein and indocyanine green angiographies in Susac syndrome. Retina. 2007;27(9): 1238-42.

9. Susac JO. Susac syndrome. Am J Neuroradiol. 2004;25: 351-2.

10. Clément P, Conessa C, Dot C, Kossowski M, Flocard F, Poncet JL. Susac syndrome or microangiopathy of the cochlea, brain and retina. Ann otolaryngol Chir Cervicofac. 2003; 120(1):4953.

11. Rennebohm RM, Susac JO. Treatement of Susac's syndrome. JNS. 2007;257:215-20. 